\title{
Note sur les messages de végétaux : quelques exemples océaniens
}

\section{Michel Aufray}

\section{(2) OpenEdition}

1 Journals

Édition électronique

URL : https://journals.openedition.org/jso/1546

DOI : $10.4000 /$ jso. 1546

ISSN : $1760-7256$

Éditeur

Société des océanistes

\section{Édition imprimée}

Date de publication : 1 décembre 2002

Pagination : 223-227

ISSN : 0300-953x

\section{Référence électronique}

Michel Aufray, « Note sur les messages de végétaux : quelques exemples océaniens », Journal de la Société des Océanistes [En ligne], 114-115 | Année 2002, mis en ligne le 26 mai 2008, consulté le 09 mars 2022. URL : http://journals.openedition.org/jso/1546 ; DOI : https://doi.org/10.4000/jso.1546

\section{(c)}

Journal de la société des océanistes est mis à disposition selon les termes de la Licence Creative Commons Attribution - Pas d'Utilisation Commerciale - Pas de Modification 4.0 International. 


\title{
Note sur les messages de végétaux : quelques exemples océaniens
}

par

\author{
Michel AUFRAY *
}

\section{RÉSUMÉ}

Dans les sociétés océaniennes, les messages de végétaux sont communément employés dans diverses circonstances sociales pour transmettre une information. Le sens à véhiculer peut être motivé par l'aspect de la plante ou bien, comme sur l'île Anatom (Anecom), au Vanuatu, les messages peuvent être élaborés à partir d'un principe phonétique: le nom du végétal évoque l'idée à transmettre.

Mots-CLÉs: Vanuatu, Anatom, ethnobotanique, sémiologie.

La réalité langagière d'une culture ne concerne pas seulement la communication linguistique ; elle recouvre aussi les modes de communication non verbaux, ceux-ci pouvant utiliser divers supports : langage du corps, objets, marques, icônes et signes. Leur existence dans les sociétés océaniennes a souvent été signalée mais, généralement, ces systèmes d'information n'ont suscité qu'un simple intérêt documentaire. Ils mériteraient à notre avis d'être inventoriés et étudiés car ils participent aux échanges sociaux au sein d'une communauté.

Les messages de végétaux, en particulier, tiennent un rôle non négligeable. À la différence de la communication verbale, ils permettent de transmettre une information sans limitation de temps et d'espace.

\begin{abstract}
In Oceanian societies, plant messages are commonly used in different social circumstances to pass on information. The meaning to convey may be motivated by plant appearance or, as on Aneityum island in Vanuatu, messages may be elaborated from a phonetic principle: the name of the plant summons to the mind the word of sentence to understand.
\end{abstract}

Keywords : Vanuatu, Aneityum, ethnobotany, semiology.

\section{Les messages " carte de visite »}

On a recours à ce moyen de communication lorsque les interlocuteurs sont éloignés les uns des autres et que l'échange verbal est impossible ou bien lorsque quelqu'un veut laisser une trace de son passage en un lieu. Ces messages de plantes fonctionnent alors comme des « cartes de visite ». Naguère, ils étaient en usage en Nouvelle-Calédonie, dans la région de Houailou ${ }^{1}$. On les appelait kavië, mot qui signifie «qui est aimable » et qui désigne aussi un " insecte du genre Elatnydae, qui penche la tête et le thorax en avant, d'un coup sec, comme s'il saluait » (Leenhardt, 1935 : 139). Ces kavië pouvaient être individuels ou collectifs. Ils permettaient de connaître les déplacements des person-

* Professeur à l'Institut national des langues et civilisations orientales, Paris.

1. Les informations concernant la région de Houailou nous ont été amicalement communiquées par Jacqueline de La Fontinelle. 
nes ou, déposés sur le seuil de la maison, ils informaient de la venue de visiteurs. C'est ainsi qu'au village de Ba près de Houailou, il y avait des kavië à plusieurs sorties du village. Chaque habitant, en revenant des jardins, jetait négligemment une plante qui était reconnaissable par les voisins. Le degré de fraîcheur du végétal indiquait même quand son propriétaire était passé.

\section{Les marques de souvenir, de serment, de propriété ou d'interdit}

On utilise aussi des végétaux lorsque la transmission d'une information nécessite un moyen d'expression permanent, par exemple, pour valider un serment, mémoriser un événement, marquer une appartenance ou un interdit. Le message peut être matérialisé par des branches cassées, des encoches sur les troncs d'arbre, des signes gravés sur les végétaux. Maurice Leenhardt rapporte ainsi que pour marquer sa propriété sur une plantation :

« La femme qui plante un taro trace un signe qui lui est propre au bas du tubercule. Lorsque, deux ou trois ans après, on arrache le taro, le signe est demeuré sous la racine, et la propriété de la plante reste incontestée » (Leenhardt, 1947 : 244-245).

Les plantes nouées mais non déracinées, souvent attachées à une perche, signalent généralement des interdits : lieu de pêche réservé, jardin à ne pas traverser, nourriture à ne pas consommer, etc.

Au Vanuatu, sur l'île Anecom ${ }^{2}$, l'extrémité d'un roseau est nouée pour signifier aux femmes et aux enfants qu'ils doivent s'éloigner de la place où les hommes boivent le kava. À Houailou, pour indiquer qu'il est défendu de récolter de la nourriture sur une propriété, on place des interdits, mââyöö, qui sont généralement faits d'une touffe d'herbe non arrachée et nouée. Ils peuvent aussi être confectionnés avec des végétaux attachés à une perche ou avec une palme de cocotier nouée sur elle-même.

À Maré, aux îles Loyauté, co tha cebul signifie « jeter l'interdit sur»:

«On plante une grande perche dans l'endroit interdit avec un échantillon de l'objet défendu. Ainsi sur un rivage, si le maître veut garder l'herbe pour son usage et éviter qu'on ne la brûle, il attache une gerbe à environ un mètre du sol » (Dubois, 1981 : 17).
À Houailou, pour avertir qu'une femme avait ses règles, on plaçait devant sa case une feuille de bananier sèche (joojo).

\section{Les messages secrets}

Ce procédé est également employé lorsque l'information à véhiculer est à diffusion restreinte ou doit rester secrète, ainsi les messages échangés entre amoureux. Naguère, en Nouvelle-Calédonie, les garçons et les filles qui ne devaient pas se montrer ensemble en public se fixaient des rendez-vous discrets en plaçant sur les chemins des plantes comme signes de reconnaissance. Georges Henri Luquet a décrit cette pratique :

«Les fleurs et les branches sont également employées par les Canaques, d'une façon curieuse pour leurs déclarations d'amour. Le canaque amoureux creuse sur le chemin de sa tribu un trou large et peu profond; il y dépose des fleurs, des feuilles et continue sa route. Si le soir, il voit dans le village à la main d'une femme des feuilles ou des fleurs semblables à celles qu'il a mises sur le chemin, il sait que cet amour est partagé » (Luquet, 1926 : 4).

À Maré, ces messages s'appellent ae-len, " pâté de chemin »:

«On met sur le bord du chemin des feuilles dont le nom spécifique commence par le même son que le nom propre de sa personne. C'est un signe pour prévenir ceux qui vous suivent de votre passage, pour voir la direction que vous avez suivie... Le jeune homme déposait son ae-len, sur lequel la fille séduite posait à son tour, ses propres feuilles » (Dubois, 1981 : 17).

On peut penser que c'est par souci de préserver une certaine confidentialité que pour les fêtes, à Tanna, au lieu de commander oralement un chant aux compositeurs, on leur présente des paquets ou des paniers de plantes. Après les avoir examinés, ils en dégagent alors un thème d'inspiration (Bonnemaison, $1986: 476$ ).

\section{Les messages, annonces d'événements graves}

La littérature orale mélanésienne fait parfois allusion à des plantes utilisées comme signes pour aviser d'une mauvaise nouvelle. Ainsi, dans un récit en langue paicî, Le chef de Göbwînyârâ (Guiart, 1957 : 61-65) ${ }^{3}$, le héros avant de partir

2. Les cartes mentionnent Anatom ou Aneityum. Les informations concernant cette île ont été recueillies lors d'enquêtes que nous avons effectuées en 1977 et 1978. Nous adopterons ici les conventions graphiques suivantes : <c> note l'occlusive palatale sourde/c/, $<\mathrm{g}>$ la nasale vélaire $/ \eta /,<\mathrm{d}>$ la spirante interdentale sourde $/ \theta /,<\mathrm{x}>$ la spirante vélaire sonore $/ \gamma /$.

3. Une autre version inédite en langue a’jië a été recueillie par J. de La Fontinelle. 
en voyage, attache au toit de sa maison, une touffe de méâmöru (Plectranthus parviflorus Henck., Labiées) et s'adresse ainsi à son cadet :

« Je jette sur le toit de la maison une liane méâmöru, tant qu'elle sera verte, c'est qu'il ne m'arrivera que de bonnes choses ; si elle crève, c'est que j'aurai rencontré un malheur ».

À Houailou, la fougère bwâwè (Pteridium aquilinum Kuhn, Dennstaedtiacées), repliée le long d'un chemin, annonçait un événement grave (Leenhardt, 1935 : 58). Un morceau de branche de kârhé (Araucaria cookii R. Br., Araucariacées) accompagnait les messages de deuil. Une petite igname y était ajoutée. On la présentait à la personne qui était chargée de préparer les vivres pour la fête de deuil (pilou). Si la tête du tubercule était cassée, cela voulait dire que le pilou n'aurait lieu que l'année suivante, car la première récolte serait bouturée et replantée pour obtenir une multiplication des ignames. Les cannes à sucre servaient aussi de « carton d'invitation » lors des fêtes. Un fragment de canne à sucre dont la pointe terminale était conservée impliquait que la fête serait importante : toutes les nourritures seraient récoltées et mangées et l'on détruirait ensuite tous les biens du mort. Sur Anecom, pour annoncer les deuils, on cassait le germe d'une noix de coco puis on perçait l'un des « yeux » de la noix ce qui voulait dire : « Mon œil est crevé, quelqu'un de ma famille est mort ».

Dans la langue a'jië, le terme bùrù désigne les objets servant de correspondance de guerre ou de signe d'alliance ou de rupture. À cet effet, des nœuds d'écorces de awa' (Broussonetia papyrifera (L.), Beauv., Moracées) ou de aree (niaouli, Melaleuca leucadendron L., Myrtacées) étaient en usage comme le montre une des planches d'illustrations des Notes d'Ethnologie néocalédonienne de Maurice Leenhardt. Chaque type de nœud correspondait à un sens différent : assassinat, alliance, déclaration de guerre, paix... (Leenhardt, 1930 : pl. XXIII).

À Maré, des messages en nœuds de tapa, i-rekaica $^{4}$, étaient également employés pour établir des alliances guerrières ou les renverser (Dubois, 1981: 18).

Les plantes arrachées ou coupées sont généralement symboles de deuil ou de guerre ${ }^{5}$. Ainsi, dans les chants anecom, la métaphore de la fleur d'hibiscus cassée est souvent employée pour évoquer la mort ou le conflit. À Houailou, lors des guerres, les touffes arrachées de l'herbe dèo (Imperata cylindrica (L.), Graminées) annonçaient la destruction totale des attaqués.

On retrouve dans d'autres régions océaniennes l'utilisation de végétaux pour signifier l'établissement d'alliances ou, au contraire, le déclenchement des hostilités. Quand les habitants de l'̂̂le de Pukapuka croisaient des bateaux étrangers, ils leur lançaient la nervure centrale d'une palme de cocotier, appelée kaleva manu (oiseau kaleva, coucou tahitien, Urodynamis taitensis Sparrman) ou yiliyili « question ». Si les étrangers la conservaient, ceci était un signe de paix. S'ils la renvoyaient, cela signifiait que leurs intentions étaient belliqueuses. Si deux autres nervures étaient expédiées avant le retour de la première, il s'agissait d'une déclaration de guerre (Beaglehole, 1938 : 374).

James Morrison rapporte qu'à Tahiti, des branches, appelées rauava, étaient utilisées par les messagers.

«Lorsqu'un messager est dépêché avec une telle délégation, il donne une feuille à chaque personne auprès de laquelle il est envoyé et celle-ci, après avoir reçu cette feuille et pris connaissance du nom du chef qui l'envoie ne met jamais sa parole en doute » (Morrison, $1981: 198)$.

\section{Les messages à motivation linguistique}

Dans les exemples rapportés ci-dessus, le choix de la plante est soit motivé par l'aspect de la plante, soit par une caractéristique fonctionnelle. Mais le message peut fonctionner aussi comme un support transposé du langage. Il s'agit dans ce cas du procédé appelé par Marcel Cohen, rébus à transfert de choses : le nom de la plante évoque l'idée à transmettre (Cohen, $1958: 15)$.

Des messages de ce type sont en usage sur l'île Anecom où ils sont désignés par le terme générique de netupu. Le principe consiste à associer le nom d'une plante et, parfois, d'un minéral ${ }^{6}$, avec un autre terme du lexique qui lui est homophone ou quasi-homophone, le sens de ce mot étant en rapport avec l'information à transmettre. Ainsi, dans cette langue, la cordyline (Cordyline fruti$\operatorname{cosa}$ (L.) Chevalet, Liliacées) se dit inrowod. Ce terme est rapproché de awod qui signifie « battre, frapper à mort quelqu'un ». Si on place une feuille de cordyline devant la maison de quelqu'un, on déclare son intention de le tuer. Le lien entre le signifiant et le signifié repose donc

4. De nos jours, ce mot a le sens d'espion.

5. C'est pourquoi, naguère, il était inconvenant d'offrir des fleurs coupées à des Mélanésiens car elles évoquent la mort.

6. Le monde minéral et le monde végétal sont souvent confondus. Les rochers ont ainsi une croissance comparable à celle des plantes. 
sur une analogie phonique, une sorte de calembour, un peu comme si en français, une feuille de frêne était placée sur un panneau routier pour inviter les automobilistes à «freiner».

Pour la confection de ces messages, on emploie les branches, les fleurs, les feuilles mais aussi les fruits. Ainsi, l'arbre à pain (Artocarpus altilis Forst, Moracées) se dit inma, mot qui désigne aussi le deuil. C'est pourquoi les fruits d'arbre à pain étaient naguère en usage pour annoncer les décès. Il existe aussi une variété de noix de coco inxopwou dont on mâche la bourre sucrée. Xopwou veut dire «bon, aimable». La noix de coco inxopwou servira donc à évoquer un homme affable et généreux.

On observe que les termes mis en correspondance ne sont pas toujours en relation d'homophonie complète. Le plus souvent, le rapprochement ne s'effectue que sur la similitude d'une seule syllabe comme dans les exemples qui suivent :

Pour signaler un droit de pêche, on placera près d'un trou d'eau du récif, des branches de nawa (Polyscias scutellaria) qui rappelle le mot owa / ohowa "plein, rempli », d'où le sens du message : "Ce trou d'eau est plein de poissons, la pêche y est prohibée ». De même, l'arbre nefitau (Ficus edulis Bur., Moracées) est associé à taupan, terme qui veut dire «s'appliquer à, dépendre de, concerner ». Les feuilles de nefitau ont alors pour sens: "Mêle-toi de ce qui te regarde ». Un autre arbre, inmop (Inocarpus edulis Forster, Légumineuses papilionacées) est associé à atumop "se reposer». Si un visiteur dépose des feuilles ou des fruits de cet arbre devant la demeure de quelqu'un qui est absent, cela signifiera : " Je suis venu te voir. Tu n'étais pas là. Je me suis reposé chez toi durant ton absence ».

Il est possible de réunir plusieurs plantes ensemble dans un même message. On obtient alors un énoncé complet combinant plusieurs signifiés. Ainsi, l'arbre tamanou (Calophyllum inophyllum L. Guttifères) se dit inpexe. Ce nom rappelle un autre mot, inpeke, qui signifie « terre, pays ». Les feuilles de tamanou sont utilisées en association avec celles d'un arbuste nida (Decaspermum sp.). Ce nom est rattaché à eda qui est la particule interrogative « où? ». La combinaison inpeke + nida se traduira alors par : « Où est ton pays? D'où viens-tu ?». Cette question sousentend que l'individu à qui ce message est adressé se trouve sur une terre étrangère où sa présence est indésirable. Une autre plante naha désigne une sorte de lis de bord de mer (Crinum asiaticum L. Amaryllidacées). Naha est relié à aha «fuir, s'enfuir». On aura ainsi : inpexe + naha qui voudra dire « Retourne chez toi sur ta terre!»

Le nom de la plante renvoie parfois à un toponyme. On a ainsi l'arbre kaliteg (Cerbera odollam Gaetn., Apocynacées) qui est mis en correspondance avec le village d'Iteg ou encore, l'arbre inmec (Trichospermum sp.) avec le village d'Umec au sud de l'île. Si l'on dépose les feuilles de ces trois végétaux : naha + inpexe + inmec sur le chemin que doit emprunter un individu, on lui notifie alors qu'il se trouve sur une propriété qui n'est pas la sienne et qu'il doit retourner chez lui à Umec.

À un même végétal peut être attaché plusieurs significations. C'est ainsi qu'un arbuste grimpant appelé name (Freycinetia sp., Pandanacées) évoquera soit le village de Aname, un toponyme situé au nord de l'île, soit le mot ame "vide, aveugle ». Dans ce dernier cas, le message voudra dire : «Tu es aveugle », sous-entendu : « Tu as fait des choses que tu n'aurais pas dû faire ».

Ces messages avaient parfois une portée politique. Les gens d'Anecom racontent avoir employé autrefois un message comportant des feuilles de l'arbre nametayeg (arbre non identifié) à l'encontre d'un homme qui voulait être chef. Dans ce cas, nametayeg renvoyait au mot ayeg « tirer vers soi », ce qui laissait entendre que cet homme voulait «tirer» les honneurs à lui mais qu'il n'était pas digne de la fonction convoitée.

\section{Médicaments et magies}

Ce système de rébus ne sert pas seulement à coder les messages, mais il justifie aussi certaines pratiques magiques et médicinales. Par exemple, lorsqu'on traversait l'île, on prenait une feuille de la liane napwotet (liane non identifiée). On la pliait en deux et on la conservait sur soi. La distance à parcourir était ainsi réduite de moitié, napwotet étant rapproché de upwotpotet "près de, à côté de, à proximité de ».

Par le terme nenah, on désigne un état de troubles psychologiques provoqué par le départ d'un ami que l'on a hébergé. Cette absence a pour conséquence d'attirer la malchance sur la personne restée seule : mauvaises pêches, récoltes infructueuses. Pour mettre un terme à ces effets néfastes, on prend une feuille de naha ( $\mathrm{Cri}$ num asiaticum. L. Amaryllidacées) dans laquelle on enveloppe une pierre. On se passe ensuite ce paquet autour du corps puis on le lance en l'air. Par ce geste, on « chasse » le mauvais sort, ceci en vertu de la similitude phonique entre naha et aha « fuir, s'enfuir ».

Inpa est un arbuste aux feuilles odoriférantes (Euodia hortensias) qui servent à confectionner 
les couronnes de danse. Elles sont également employées dans la magie pour provoquer la tempête en mer ou, à l'inverse, ramener le calme. On édifie sur le rivage un petit tas de pierres au sommet duquel on place des feuilles de inpa. Sur celles-ci on dépose un caillou. Lorsqu'on retire ce dernier, la mer se déchaîne; quand on le replace, les flots s'apaisent aussitôt. Dans ce cas, le choix des feuilles de l'arbuste inpa est dicté par le rapprochement avec le mot inypa «être en paix, être calme ».

L'emploi du principe phonétique dans l'élaboration de ces messages constitue donc une transition entre l'objet et la parole, ce qui est un premier stade dans le processus d'objectivation du langage. Ce procédé joue aussi un rôle dans la construction du symbolisme culturel : les rapports qui lient le symbole non linguistique à son signifié peuvent être fondés sur une analogie morphologique ou fonctionnelle entre la chose signifiante et la chose signifiée ou bien, comme dans le cas de ces messages de végétaux, la langue peut intervenir directement dans la formation du symbolisme, le lien de signifiant à signifié reposant alors sur l'analogie lexicale.

\section{RÉFÉRENCES}

Beaglehole, E. et P., 1938. Ethnology of Pukapuka. Honolulu : Bernice P. Bishop Museum.

BonnemaIson, J., 1986. Les fondements d'une identité. Territoire, histoire, et société dans l'Archipel de Vanuatu (Mélanésie): Tanna, les hommes lieux, tome II. Paris : ORSTOM.

CoHen, M., 1958. La grande invention de l'écriture et son évolution. Paris: Imprimerie nationale, C. Klincksieck.

DuBoIs, M. J., 1981. La communication par signes à Maré, îles Loyauté. Bulletin de la Société historique de Nouvelle-Calédonie 47 : 13-19.

Guiart, J., 1957. Contes et légendes de la GrandeTerre. Nouméa : Éditions des Études mélanésiennes.

LeEnHARDT, M., 1930. Notes d'ethnologie néocalédonienne. Paris : Institut d'ethnologie.

-, 1935. Vocabulaire et Grammaire de la langue houaïlou. Paris : Institut d'ethnologie.

_, 1947. Do Kamo, la personne et le mythe dans le monde mélanésien. Paris : Gallimard.

Luquet, G. H., 1926. L'Art néo-calédonien. Paris : Institut d'Ethnologie.

Morrison, J., 1981. Journal de James Morrison, second maître de la Bounty. Papeete : Société des Études océaniennes. 